\title{
Sistema para a Geração Automática da Escrita de Sinais em SignWriting visando o Apoio ao Ensino e à Aprendizagem da Libras
}

\author{
Carlos Eduardo Iatskiu ${ }^{1}$, Laura Sánchez García ${ }^{1}$, Tanya Amara Felipe ${ }^{2}$ \\ e Diego Roberto Antunes ${ }^{3}$
}

\author{
${ }^{1}$ Universidade Federal do Paraná (UFPR) - Curitiba, PR \\ ${ }^{2}$ Instituto Naciona de Educação de Surdos (INES) - Rio de Janeiro, RJ \\ ${ }^{3}$ Universidade Tecnológica Federal do Paraná (UTFPR-PG) - Ponta Grossa, PR \\ ceaiatskiulinf.ufpr.br, sg.laura, tanyafelipe, drantunes egmail.com
}

\begin{abstract}
Brazilian Deaf communities face problems within their teaching/learning processes mainly because of the lack of written courseware in their preferential language, Libras (Brazilian Sign Language). Sign Writing is a writing system that partially solves this problem, but the interaction with the sign writing tool is slow and does not stimulate its appropriation by the target communities. This paper describes a system that allows for sign automatic generation from its specification in a phonological model that diminish the problem space.
\end{abstract}

Resumo. As comunidades Surdas do Brasil enfrentam problemas nos seus processos de ensino/aprendizagem, principalmente devido à falta de material escrito em sua língua preferencial, a Libras (Língua Brasileira de Sinais). O SignWriting é um sistema de escrita que resolve parcialmente este problema, mas a interação com a ferramenta para a escrita de sinais é lenta e não estimula a sua apropriação pelas comunidades alvo. Este artigo descreve um sistema que permite a geração automática de sinais a partir de sua especificação num modelo fonológico que diminui o espaço do problema.

\section{Introdução}

A escrita é um pré-requisito para a formação do ser humano, além de ter dado ao homem a possibilidade de expandir suas mensagens além do seu próprio tempo e espaço, pois é a porta de entrada para a cultura, saber tecnológico, científico, entre outros [Garcez 2018]. A aquisição de habilidades de escrita na Língua Portuguesa pelos Surdos exige processos de ensino/aprendizagem e metodologias específicas semelhantes aos processos de aprendizado de uma língua estrangeira para os ouvintes. A língua de sinais do país é a língua preferencial para os Surdos, sendo considerada, linguisticamente, sua primeira língua, embora possa não ser a primeira do ponto de vista cronológico.

A Língua Brasileira de Sinais (Libras) é o sistema linguístico legítimo e natural utilizado pela Comunidade Surda Brasileira que possibilita o desenvolvimento linguístico, social e intelectual do indivíduo que a utiliza como instrumento comunicativo, favorecendo seu acesso ao conhecimento cultural-científico, bem como a integração ao grupo 
VII Congresso Brasileiro de Informática na Educação (CBIE 2018)

Anais do XXIX Simpósio Brasileiro de Informática na Educação (SBIE 2018)

social ao qual pertence. Em nosso país, a Libras foi oficializada como a língua dos Surdos com o decreto de Lei número 10.436/2002, que garantiu direitos como o acesso à informação em Libras nas instituições de ensino e a comunicação em processos seletivos, transformando-a em uma disciplina obrigatória em cursos de graduação e formação de professores [Antunes 2011].

Embora as línguas de sinais sejam de modalidade gestual-visual, diversos sistemas de escrita e transcrição surgiram para solucionar a grafia dos sinais, como o Sistema de Stokoe e a Notação de François Neve[Stumpf 2000], HamNoSys [Prillwitz et al. 1989], ELIS [Barros 2008] e o SignWriting [Sutton 2013b]. Este último vem sendo utilizado por diversas instituições de todo o mundo e tem um poder significativo de representação do qual a sua complexidade decorre. A escrita por meio do SignWriting expressa: configurações de mãos, movimentos, expressões faciais e pontos de articulação das línguas de sinais. Já são mais de 35 os países que utilizam o sistema de SignWriting em escolas, universidades, associações e áreas ligadas às Comunidades Surdas [Sutton 2013a].

Diversos estudos sobre a análise de produções textuais dos Surdos apontam que a escrita dos Surdos não segue as mesmas construções dos sistemas de escrita para ouvintes, que se apoiam na linguagem oral para a produção textual. Assim, muitos Surdos possuem dificuldades na aprendizagem da leitura e da escrita do Português [Guarinello 2005]. Na literatura existente, são encontradas ferramentas que têm o intuito de fornecer apoio aos Surdos que desejam utilizar o SignWriting. No entanto, essas ferramentas apresentam barreiras que, acreditamos, possam contribuir com o baixo uso pelos usuários Surdos, principalmente o excesso de tempo despendido para a construção de um único sinal e a necessidade de os sinais estarem dicionarizados para sua grafia, pela falta de um sistema inteligente para a sua geração [Iatskiu et al. 2015]. Como trabalhos relacionados é possível citar as seguintes ferramentas: AGA-Sign, SignNet, SW-Edit, SignTalk, SignSim, SignEd, SignWriter, SignPuddle, SignWebMessage e SignWriter Studio.

Com o intuito de apoiar a Comunidade Surda Brasileira na aquisição de sua cidadania plena, foi criada a Arquitetura Computacional para a Interação HumanoComputador em Língua de Sinais (HCI-SL). A HCI-SL tem o intuito de proporcionar e desenvolver um ambiente integrado de ferramentas computacionais a partir de um conjunto de hipóteses e estratégias metodológicas com potencial de tratar a Libras de forma correta tanto do ponto de vista linguístico como computacional [García et al. 2013]. Essa arquitetura possui um módulo central, conhecido como: CORE-SL - Modelo Computacional para Representação de Sinais em uma Arquitetura de Serviços HCI-SL, que tem a capacidade de representar, em princípio, os sinais de qualquer língua de sinais existente por meio de um formalismo, ou seja, regras formais. Desta maneira, proporciona a base para o desenvolvimento de frameworks e ferramentas que tratam questões específicas para a construção de aplicações em línguas de sinais, dentre as quais está a escrita em SignWriting [Antunes 2015].

Com base em estudos do problema da escrita enfrentado pelas Comunidades Surdas, da análise das ferramentas existentes e da base da Arquitetura HCI-SL, foi identificado o espaço para o desenvolvimento de uma ferramenta de geração automática dos sinais da Libras e, potencialmente, de qualquer língua de sinal usada ao redor do mundo, de maneira a tornar a escrita uma tarefa mais simples para os Surdos. O presente artigo apresenta detalhes do desenvolvimento e dos testes do módulo da Arquitetura HCI- 
VII Congresso Brasileiro de Informática na Educação (CBIE 2018)

Anais do XXIX Simpósio Brasileiro de Informática na Educação (SBIE 2018)

SL chamado Gerador de SignWriting, que proporciona uma solução para o problema em questão. Esta ferramenta realiza a leitura das especificações dos sinais da Libras no CORE-SL e faz a geração dos sinais correspondentes em SignWriting.

O gerador tem aplicação direta no contexto de ensino/aprendizagem do SignWriting junto às comunidades Surdas, podendo, também, funcionar como ferramenta de consulta durante a atividade de escrita. Cabe destacar que os usuários potenciais não se restringem aos Surdos, mas se estendem aos profissionais que atuam junto a essas comunidades. Adicionalmente, os resultados esperados com a aplicação deste trabalho, na prática, consistem na geração de ferramentas para apoiar as Comunidades Surdas na criação de conteúdos e de materiais didáticos na forma escrita, em SignWriting.

\section{Bases Conceituais}

Nesta seção são apresentadas três bases metodológicas do trabalho em questão: Arquitetura HCI-SL, Modelo de Representação CORE-SL e Sistema de Escrita SignWriting.

\subsection{Arquitetura HCI-SL}

A ferramenta objeto do presente artigo consiste em um módulo da Arquitetura Computacional para a Interação Humano-Computador em Língua de Sinais. A HCI-SL foi criada para integrar hipóteses, estratégias metodológicas e serviços capazes de resolver, conjuntamente, o problema do tratamento computacional da Língua Brasileira de Sinais e, por consequência, auxiliar na quebra da barreira social de acesso à informação e ao conhecimento enfrentada pelas comunidades de Surdos [García et al. 2013]. Essa Arquitetura é composta de três camadas:

1. API Interna: camada responsável por disponibilizar os serviços computacionais fundamentais (reconhecimento automático de sinais, processamento de SignWriting, tradutores, agentes virtuais 3D e outras).

2. API de Serviços: camada responsável por recursos computacionais que utilizam os serviços da camada interna de API (dicionários, tradutores, vocabulários controlados, ambientes virtuais de comunicação, etc).

3. Aplicações: camada que se refere aos aplicativos para o usuário final, entre os quais jogos e sistemas de apoio ao letramento.

O Gerador descrito neste artigo se insere na segunda cama. O esquema apresentado na figura 1 representa as relações específicas entre os serviços da Arquitetura HCI-SL, o módulo central CORE-SL e o Gerador de SignWriting, destacado.

\subsection{Modelo Computacional CORE-SL}

O Modelo Computacional CORE-SL[Antunes 2015] foi construído com base nos sistemas existentes na linguística e na escrita das línguas de sinais, e na codificação computacional, de modo a viabilizar o tratamento de questões fundamentais como a verificação da forma correta na representação (por meio de regras), a complexidade do armazenamento e a recuperação, a similaridade (capacidade de distinguir computacionalmente sinais muito semelhantes), funcionar como base para o desenvolvimento de frameworks que sirvam de base para a construção de aplicações em línguas de sinais, entre outras. É composto por quatro camadas principais: 
VII Congresso Brasileiro de Informática na Educação (CBIE 2018)

Anais do XXIX Simpósio Brasileiro de Informática na Educação (SBIE 2018)

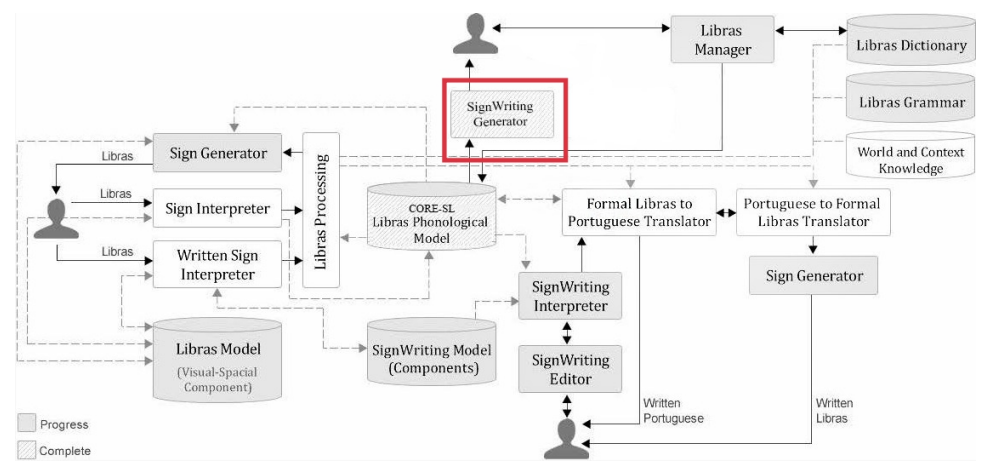

Figure 1. Esquema da Arquitetura HCl-SL - Adaptado de: [García et al. 2013]

1. Interna: permite o armazenamento e acesso aos sinais. Trabalha com a indexação, a escalabilidade, a segurança e o desempenho (tratamento computacional).

2. Conceitual: especifica a relação entre os modelos conceitual e formal, descreve toda a estrutura e as regras necessárias à representação dos sinais da camada.

3. Externa: apresenta os dados aos usuários em uma API que possibilita o uso das aplicações da Arquitetura HCI-SL, e operações básicas (inserção, e formatação da saída) dos sinais.

4. Uso: aplicação específica do CORE-SL para auxiliar no processo de desenvolvimento de ferramentas da Arquitetura.

Além de ser base para o desenvolvimento do Gerador de SignWriting que está sendo apresentado neste trabalho, o CORE-SL atua como pedra fundamental de outros trabalhos, entre o quais o Reconhecimento das Configurações de Mão da Libras por Malhas 3D [Porfirio 2013] e o Avatar 3D para a Síntese de Sinais da Libras [Gonçalves 2013], e ainda, o Dicionário da Libras e o Modelo Computacional da Morfologia da Libras, ambos em desenvolvimento.

\subsection{Sistema de Escrita SignWriting}

Os componentes do SignWriting permitem que as línguas de sinais sejam representadas de forma gráfica de maneira bastante semelhante (pela correspondência entre os componentes dos sinais interpretados e os símbolos do SW) à forma de interpretação dos sinais. O sistema foi desenvolvido pela norte-americana Valerie Sutton, na década de 70, quando a pesquisadora estava na Universidade de Copenhague, Dinamarca, grafando balés tradicionais a partir de um sistema criado para essa finalidade, o DanceWriting [Sutton 2013b]. Hoje já são mais de trinta e cinco países que utilizam o SignWriting em escolas, universidades, associações e áreas ligadas às comunidades Surdas [Sutton 2013a].

Segundo [Capovilla and Raphael 2001], o SignWriting objetiva ser mais que um sistema de notação científica de descrição de sinais, tendo como intuito ser um sistema prático para a escrita dos sinais, possibilitando a comunicação escrita rápida e inequívoca por Surdos em seu cotidiano. O SignWriting é robusto e capaz de representar graficamente qualquer língua de sinais, atuando como sistema alfabético cujas unidades gráficas correspondem às unidades que formam os sinais [da Rocha Costa and Dimuro 2003]. O SignWriting possui uma estrutura composta de quatro elementos básicos: mãos, movimentos, expressões faciais e corpo. Devido à modalidade gestual-espacial das línguas 
VII Congresso Brasileiro de Informática na Educação (CBIE 2018)

Anais do XXIX Simpósio Brasileiro de Informática na Educação (SBIE 2018)

de sinais, componentes tais como as expressões faciais e os movimentos corporais são imprescindíveis para uma compreensão correta de um sinal, e o SignWriting possibilita representar na escrita todas estas características minimizando a perda de detalhes.

Por outro lado, o poder expressivo do SignWriting determina a sua complexidade. Fazendo uma analogia de suas primitivas com as letras que tem a mesma função na formação das palavras nas línguas orais, enquanto o alfabeto da Língua Portuguesa tem 26 letras, o SignWriting tem mais de 10.000 primitivas. Neste contexto, tem-se a hipótese de que somente o uso da tecnologia de forma adequada pode auxiliar as Comunidades Surdas no acesso à informação e ao conhecimento na forma escrita das línguas de sinais.

\section{Gerador Automático}

O processo de desenvolvimento da ferramenta foi iniciado com um amplo estudo do Modelo Computacional CORE-SL e do SignWriting, que são, respectivamente, a entrada e a saída da ferramenta. Em paralelo, foi realizada uma revisão das ferramentas existentes na literatura que possuem suporte ao SignWriting, com o objetivo de projetar um serviço que solucione as limitações existentes no estado da arte. Após esse estudo,foram desenvolvidas, em sequência, as fases de levantamento de requisitos, projeto do software (incluindo diagramas UML e design de interface), desenvolvimento do software e testes.

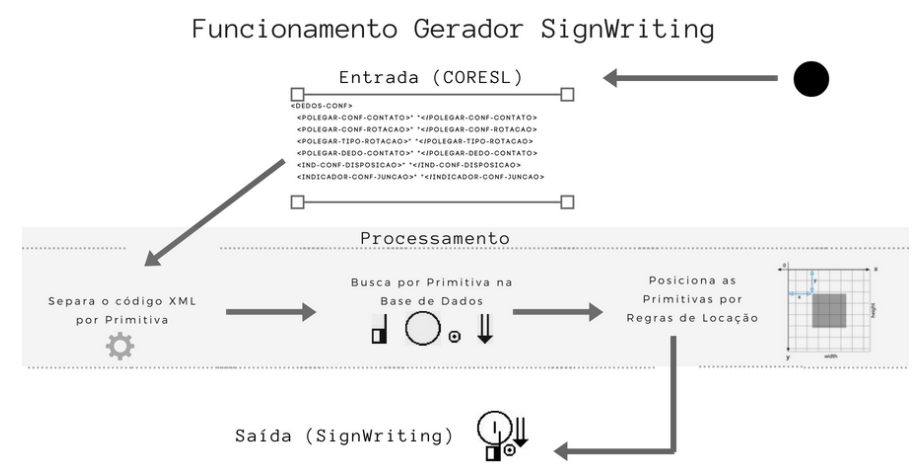

Figure 2. Diagrama do funcionamento do Gerador de SignWriting.

A ferramenta foi desenvolvida com Laravel, um framework PHP utilizado para o desenvolvimento web e para a persistência dos dados foi utilizado o Sistema Gerenciador de Banco de Dados MySQL. Para o funcionamento do Gerador, a ferramenta fornece uma estrutura em XML para que o usuário possa inserir as descrições do sinal Libras representado pelo CORE-SL. O sistema, por meio das suas bibliotecas, faz a leitura da descrição inserida pelo usuário e o tratamento de possíveis erros. Em seguida, salva a descrição de cada componente fonológico do sinal Libras. O passo seguinte da ferramenta consiste em realizar várias buscas no banco de dados para encontrar cada primitiva do SignWriting que corresponde à descrição no CORE-SL. Se encontrada, esta primitiva do SW é exibida no espaço de sinalização de acordo com sua localização em coordenadas $(\mathrm{x}, \mathrm{y})$. A figura 2 mostra um fluxograma do funcionamento da ferramenta.

\subsection{Base de Dados}

Para o funcionamento do sistema, foi necessária uma etapa de pré-processamento que consistiu na leitura e na gravação das primitivas do SignWriting (mais de 10.000) descritas 
VII Congresso Brasileiro de Informática na Educação (CBIE 2018)

Anais do XXIX Simpósio Brasileiro de Informática na Educação (SBIE 2018)

de acordo com o CORE-SL. As primitivas do SignWriting foram separadas em quatro grupos, cada qual com suas próprias características. Na tabela 1, observa-se a forma de descrição de cada grupo do SignWriting pelo modelo computacional.

Table 1. Grupos do SignWriting pelo CORE-SL

\begin{tabular}{|l|l|}
\hline Grupos do SignWriting & Estrutura do CORE-SL \\
\hline Configuração de Mão & $\begin{array}{l}\text { articulação, braço, disposição dos dedos, junção, polegar contato, polegar rotação, polegar tipo } \\
\text { rotação, polegar dedo contato, indicador disposição, indicador junção, médio disposição, médio } \\
\text { junção, anelar disposição, anelar junção, mínimo disposição, minimo junção, orientação mão, } \\
\text { orientação palma e orientação dedos. }\end{array}$ \\
\hline Expressões Não Manuais & específica, cabeça Expressão, tronco Expressão, parte superior do rosto e parte inferior do rosto. \\
\hline Movimento & $\begin{array}{l}\text { contorno, sentido, interação, contato do movimento, local, extensão, temporal, tensão, } \\
\text { velocidade, direção, direcionalidade, plano, plano movimento, vezes e frequência. }\end{array}$ \\
\hline Movimento Local & $\begin{array}{l}\text { antebraço movimento, mão movimento, dedos movimento, polegar movimento, indicador } \\
\text { movimento, médio movimento, anelar movimento, mínimo movimento, pulso dobramento e } \\
\text { pulso torcedura. }\end{array}$ \\
\hline
\end{tabular}

Cada um destes pontos da estrutura do CORE-SL pode ainda ser preenchido por valores diferentes, como no caso do tipo do contato do polegar presente nas configurações de mão, que pode ter os seguintes valores: almofada na unha, pontas, almofadas ou unha na almofada. Adicionalmente a estrutura específica das expressões não manuais pode ser preenchida com: tristeza, alegria, felicidade, sorrindo, brava, dúvida, indiferença, desconforto, afetiva (emoção), afetiva (tristeza), choro, raiva, interrogativa, confirmação (sim), confirmação (não), afirmativa, exclamativa, negativa, assentimento, decepção, preocupação e medo.

De maneira análoga, existem diversas opções pré-determinadas pelo CORE-SL de preenchimento das estruturas de configuração de mão, movimentos direcionais e movimento local. A base de dados foi preenchida, primitiva por primitiva, cada uma com sua descrição.

Com o intuito de realizar a prova de conceito da solução desenvolvida, a base de dados foi populada com 253 configurações de mão, 31 expressões não manuais, 39 símbolos de movimento e 6 símbolos de movimento local. Este sub-conjunto foi selecionado com base no site oficial do SignWriting, que apresenta as primitivas mais usadas na formação dos sinais, semelhantemente à letra "a" do nosso alfabeto, a mais frequente na formação de palavras na nossa língua.

\subsection{Parâmetro de Localização / Ponto de Articulação}

Um dos pontos mais importantes para a formação dos sinais do SignWriting é tratado pelo CORE-SL como o parâmetro de localização, que pode ser definido como o local no corpo, nas mãos ou no espaço neutro onde o sinal é articulado. Uma das complexidades da ferramenta consistiu em trabalhar com a localização, pois os parâmetros do modelo computacional precisaram ser transformados em coordenadas $(\mathrm{x}, \mathrm{y})$ para posicionar cada primitiva.

No CORE-SL, a localização é formada por pontos no corpo (como cabeça, tronco, mãos e dedos) ou no espaço (coordenadas tridimensionais). Adicionalmente, um ponto de localização/articulação ainda pode ser detalhado com parâmetros para garantir maior precisão à descrição (e.g. localização na cabeça, na região do olho, no lado dominante do indivíduo). 
VII Congresso Brasileiro de Informática na Educação (CBIE 2018)

Anais do XXIX Simpósio Brasileiro de Informática na Educação (SBIE 2018)

\subsection{Espaço de Sinalização}

O espaço de sinalização tem papel crucial na ferramenta, pois é o local no qual o sinal é exibido e que tem a função de representar o corpo humano da cintura até o topo da cabeça e ao seu redor. É neste espaço de sinalização onde são exibidas cada uma das primitivas do SignWriting responsáveis pela formação do sinal em sua respectiva posição por meio do parâmetro de localização. Para o desenvolvimento deste módulo foi utilizado o Canvas do HTML5, que permite especificar uma área da página onde se pode desenhar e reenderizar imagens, tendo funcionado como o recurso por excelência para o posicionamento das imagens das primitivas do SignWriting no espaço de sinalização.

Para posicionar elementos no Canvas deve-se considerar seu eixo de coordenadas em duas dimensões, com ponto de partida no canto superior esquerdo, podendo-se situar os elementos a partir de qualquer ponto do Canvas com as coordenadas (x,y), sendo que $x$ cresce segundo os pixels para a direita e y com os pixels para baixo. A Figura 3(a), apresenta a geração do sinal em SignWriting referente ao sinal da Libras "surdo", com as respectivas marcações das coordenadas no Canvas.

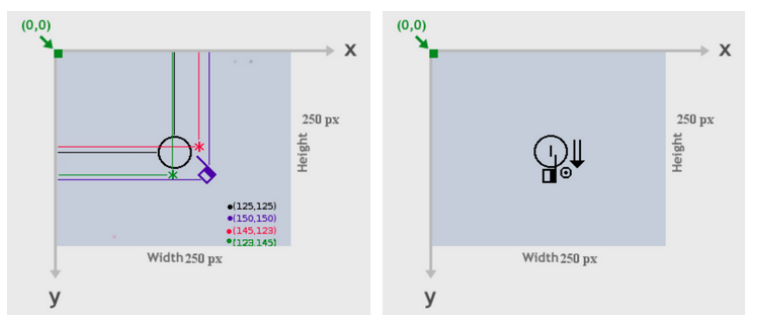

Figure 3. (a) Sinal Surdo - (b) Sinal Amarelo

Um ponto importante da utilização desta tecnologia para o desenvolvimento do espaço de sinalização é que o Canvas aceita a sobreposição de imagens e isso é algo muito comum na formação dos sinais. Na Figura 3(b) podemos ver a formação do sinal "amarelo" da Libras, na qual a configuração da mão está sobre a expressão não manual, pois nesse sinal a mão desliza sobre o rosto, mais precisamente em cima do nariz. $\mathrm{O}$ desenvolvimento da ferramenta foi finalizado com a implementação das funcionalidades para que fosse possível realizar o download dos sinais gerados. Na sequência foi iniciada a fase de testes.

\section{Experimentos de Avaliação}

Os testes da ferramenta foram realizados de duas formas distintas. No primeiro teste foi utilizada uma glosa da Libras de um gibi da Turma da Mônica: "Um Pegue Grande Vilão" [de Souza 2013], que aborda, por meio de uma linguagem descomplicada formas de se combater o Aedes Aegypti, o mosquito transmissor da dengue.

O segundo teste foi realizado com um grupo de sinais gerados também a partir deste gibi. Os sinais gerados pela ferramenta foram comparados, pixel a pixel, com aqueles gerados pelo SignWriting [Sutton 2013b] usando um comparador de imagens bastante simplificado, DiffImg.

\subsection{Geração da Escrita em SignWriting do Gibi da Turma da Mônica}

A transcrição em glosa da Língua de sinais usada na geração de materiais usando a ferramenta pode ser definida como uma representação semântica de sinais por meio de 
VII Congresso Brasileiro de Informática na Educação (CBIE 2018)

Anais do XXIX Simpósio Brasileiro de Informática na Educação (SBIE 2018)

unidades fonográficas derivadas de uma língua oral auditiva [Felipe 2014]. A escolha do gibi da Turma da Mônica se justifica pela sua utilização no letramento de crianças Surdas em Libras no Instituto Nacional de Educação de Surdos (INES). A Tabela 2, pode-se visualizar as três versões usadas para o teste: texto original do gibi, texto do gibi em glosa da Libras e a versão em SignWriting.

Table 2. Exemplo de trecho de experimento de avaliação.

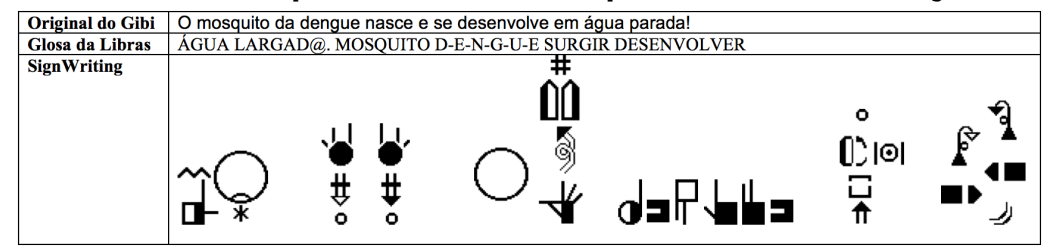

Esse texto teve como objetivo aferir se a ferramenta teria o potencial para fazer a geração dos sinais do SignWriting a partir da transcrição dos sinais da Libras através do CORE-SL. O resultado foi satisfatório, visto que todo o material transcrito foi gerado e todo texto em glosa da Libras foi traduzido para o seu correspondente em SignWriting. Também foi feita a adaptação do próprio gibi da Turma da Mônica, na qual foi realizada a substituição da conversa em Português dos balões para os sinais da Libras em SignWriting. Na figura 4 pode-se observar um trecho do gibi no qual a frase em Português "Vamos combater a Dengue!" foi substituída pelos sinais em SignWriting correspondente à glosa da Libras "D-E-N-G-U-E 2sIR1s VENCER".

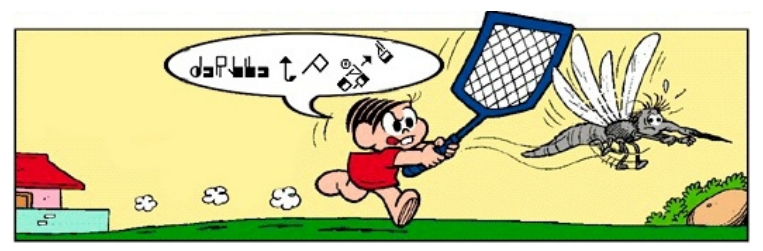

Figure 4. Trecho do gibi da Turma da Mônica em SignWriting.

\subsection{Comparação de Sinais}

O teste foi realizado por meio da comparação de 25 sinais gerados pela ferramenta, com os materiais da SignWritng. A ferramenta [DiffImg 2015] calcula a taxa de diferença entre duas imagens por meio da comparação pixel a pixel. Os resultados do teste mostraram que não houve diferença significativa entre os dois materiais, tendo apresentando um índice de similaridade acima de $97 \%$ em todos os testes. Enxergamos estes experimentos, juntamente com seus resultados, como fortes indicadores da viabilidade da futura geração de diversos materiais em SignWriting, como livros, apostilas e outro.

\section{Conclusão e Trabalhos Futuros}

O trabalho relatado neste artigo faz parte da HCI-SL, a Arquitetura Computacional para a Interação Humano-Computador em Línguas de Sinais, um ambiente integrado de ferramentas computacionais desenvolvidas a partir de um conjunto de hipóteses e estratégias metodológicas com potencial de tratar a Libras de forma correta tanto do ponto de vista linguístico quanto computacional. 
VII Congresso Brasileiro de Informática na Educação (CBIE 2018)

Anais do XXIX Simpósio Brasileiro de Informática na Educação (SBIE 2018)

Com base na HCI-SL e a partir das primitivas em SignWriting que formam um determinado sinal, geradas numa primeira etapa da pesquisa, o maior desafio enfrentado na construção da ferramenta consistiu na necessidade de completar a grafia em SignWriting com o posicionamento das primitivas no espaço de sinalização. A investigação e a escolha das tecnologias proporcionaram o suporte necessário à resolução do problema. As saídas do gerador foram testadas por meio de dois experimentos: a geração de SignWriting a partir de textos de gibis usado em contexto real na alfabetização de surdos e a comparação entre os sinais gerados pela ferramenta e a sua escrita oficial pelo órgão internacional de referência. Ambos tiveram resultados satisfatórios.

Considerando a baixa eficiência das ferramentas existentes como uma das hipóteses da não apropriação deste sistema de escrita pelas comunidades Surdas ao redor do mundo, os resultados esperados emergem naturalmente. Contrariamente às ferramentas de criação de textos escritos em SignWriting que demandam um período de tempo significativo para compor um único sinal e, assim, inviabilizam a geração de materiais longos como livros, apostilas e outros, o Gerador de SignWriting permite não somente que os sinais sejam criados mais rapidamente pelo usuário a partir de sua especificação nos componentes dos sinais, mas, também, que eles surjam de forma automática como resultado de processos de interação usuário-sistema derivados da mesma Arquitetura, entre os quais a solicitação de acesso à informação associada a um verbete da Libras e a tradução de uma palavra da Língua Portuguesa em Língua de sinais. A estatura da contribuição pode ser mensurada, ainda, a partir do fato de que mesmo ferramentas desenvolvidas pela própria organização criadora e mantenedora do SignWriting apresentam os problemas gerais relatados ou são dicionarizadas. A exigência de que cada sinal das línguas de sinais tenha seu correspondente salvo para realizar uma tradução, evidencia uma falha no potencial expressivo de suas ferramentas, tanto do ponto de vista computacional como do ponto de vista social.

O gerador tem aplicação natural no contexto real de ensino/aprendizagem do SignWriting junto às comunidades Surdas, podendo, adicionalmente, ser parte da saída de uma ferramenta de consulta durante a atividade de escrita neste sistema de escrita das línguas de sinais. Por último, considerando que diversas línguas de sinais são compostas pelo mesmo conjunto de primitivas (tanto no nível fonológico da língua quanto no conjunto de primitivas de suas grafias), conjectura-se que o CORE-SL e a ferramenta podem gerar sinais para qualquer outra língua de sinais ou, em outras palavras, são universais.

Como trabalhos futuros estão previstos o desenvolviento de outros módulos da Arquitetura, como o ambiente de consulta ao Dicionário da Libras e o Módulo SintáticoSemântico-Discursivo da Morfologia desta Língua de sinais.

\section{References}

Antunes, D. R. (2011). Um modelo de descrição computacional da fonologia da língua de sinais brasileira. Master's thesis, Pós-Graduação em Informática - Universidade Federal do Paraná, Curitiba - PR.

Antunes, D. R. (2015). Proposta de um Modelo Computacional para Representação de Sinais em uma Arquitetura de Serviços HCI-SL para Linguas de Sinais. PhD thesis, Pós-Graduação em Informática - Universidade Federal do Paraná, Curitiba - PR. 
VII Congresso Brasileiro de Informática na Educação (CBIE 2018)

Anais do XXIX Simpósio Brasileiro de Informática na Educação (SBIE 2018)

Barros, M. E. (2008). ELIS: Escrita das Línguas de Sinais: proposta teórica e verificação prática. PhD thesis, Centro de Comunicaçã£o e Expressão - Pós-Graduação em Linguística - Universidade Federal de Santa Catarina (UFSC), Florianópolis-SC.

Capovilla, F. C. and Raphael, W. D. (2001). Dicionário enciclopédico ilustrado trilingue da língua de sinais brasileira. Editora da Universidade de SÃ£o Paulo.

da Rocha Costa, A. C. and Dimuro, G. P. (2003). Signwriting and swml: Paving the way to sign language processing. Traitement Automatique des Langues Naturelles/Workshop on Natural Language Processing of Minority Languages and Small Languages, Acte of TALN 2003:193-202.

de Souza, M. (2013). "a turma da mônica - um pequeno grande vilão". http: / / turmadamonica.uol.com.br/dengue/. Acessado em 12/06/2018.

DiffImg (2015). Simple image comparison tool. https://sourceforge.net/ projects/diffimg. Acessado em 12/06/2018.

Felipe, T. A. (2014). Banco de dados para as línguas de sinais e seus sistemas de transcrição. Instrumentos linguísticos: usos e atualizações. Araruama: Editora Carolina, pages 155-188.

Garcez, T. (2018). "qual a importância da escrita?". http: / / centroevolvere. com.br/blog/qual-a-importancia-da-escrita/. Acessado em 06/06/2018.

García, L. S., aes, C. G., Antunes, D. R., and Fernandes, S. (2013). Hci architecture for deaf communities cultural inclusion and citizenship. Proceedings of the 15th International Conference on Enterprise Information Systems - ICEIS, 3:68-75.

Gonçalves, D. A. (2013). Avatar 3d para a sintese automática de sinais da língua de sinais brasileira. Master's thesis, Pós-Graduação em Informática - Universidade Federal do Paraná, Curitiba - PR.

Guarinello, A. C. (2005). Reflexões sobre a aquisição do português escrito como segunda língua de uma criança surda. Pesquisas em Linguística - Porto Alegre, 1(1):63-66.

Iatskiu, C. E. A., García, L. S., Canteri, R. D. P., and Antunes, D. R. (2015). The low use of signwriting computational tools from hci perspective. Springer International Publishing Switzerland 2015, 9176:373-382.

Porfirio, A. J. (2013). Reconhecimento das configurações de mão da libras a partir de malhas 3d. Master's thesis, Pós-Graduação em Informática - Universidade Federal do Paraná, Curitiba - PR.

Prillwitz, S., Leven, R., Zienert, H., Hanke, T., and Henning, J. (1989). Hamnosys version 2.0: Hamburg notation system for sign languages: An introductory guide. International Studies on Sign Language and the Communication of the Deaf, pages 195-278.

Stumpf, M. R. (2000). Língua de sinais: escrita dos surdos na internet. V Congresso Ibero americano de Informática Educativa.

Sutton, V. (2013a). Oficialização alfabeto internacional do signwriting. SignWriting.org.

Sutton, V. (2013b). "singwriting for sign languages". http: / / www . signwriting • org/. Acessado em 17/05/2018. 